\title{
Histone deacetylase inhibitors: A novel target of anticancer therapy (Review)
}

\author{
GREGORY KOURAKLIS $^{1}$ and STAMOS THEOCHARIS $^{2}$ \\ Departments of ${ }^{1}$ Surgery and ${ }^{2}$ Forensic Medicine, Toxicology, University of Athens, \\ School of Medical Sciences, Athens, Greece
}

Received August 25, 2005; Accepted October 17, 2005

\begin{abstract}
Accumulating evidence suggests that the acetylation and deacetylation of histones play significant roles in transcriptional regulation of eukaryotic cells. The balance between acetylation and deacetylation is an important factor in regulating gene expression and is thus linked to the control of cell fate. The histone deacetylase inhibitors (HDIs) including the hydroxamic acids, such as suberoylanilide hydroxamic acid and pyroxamide, the benzamides MS-275 and CI-994 and the butyrate derivative 4-PBA are a new class of anti-neoplastic agents currently being evaluated in clinical trials. Moreover, new synthetic HDIs have been used recently in phase I and II clinical trials. Over the next few years experts believe that as first generation HDIs produce clinical benefits and second generation inhibitors are rationally designed with improved specificity, this class of drugs will emerge as a new way of cancer treatment. The first clinical studies have shown that histone hyperacetylation can be achieved safely in humans and that treatment of cancer with such agents seems to become possible. The use of HDIs, probably in association with classical chemotherapy drugs or in combination with DNA-demethylating agents, could be promising for cancer patients. Further evaluation is needed to establish the clinical activity of combination therapy using HDIs with cytotoxic drugs or differentiation induced agents.
\end{abstract}

\section{Contents}

1. Introduction

2. HDAC inhibitors

3. Mechanisms of HDAC inhibition

4. HDAC inhibitors and anticancer therapy

5. Conclusions

Correspondence to: Dr Gregory Kouraklis, 122 Vasilisis Sofias Avenue, Athens 115 26, Greece

E-mail: gkouraklis@hotmail.com

Key words: anticancer mechanisms, anticancer therapy, cell cycle, histone deacetylase inhibitors

\section{Introduction}

Cancer is recognized as the second most common cause of death in the developed world, arising as a result of various factors. The multifactorial nature of cancer breeds a wealth of potential target genes, enzymes, proteins and hormones (1). Based on advances in cell and molecular biology, the number of therapeutic cancer targets in pipeline is rapidly increasing. Numerous long-standing pharmaceutical as well as new biotechnology companies are driving cancer research for today and future. New cancer drug delivery systems are in development to minimize harm to non-cancerous cells, whist enhancing the effect on the tumor itself.

The organization of chromatin is crucial for the regulation of gene expression. In particular, both the positioning and properties of nucleosomes influence promoter-specific transcription in response to extracellular or intracellular signals (2). Accumulating evidence suggests that the acetylation and deacetylation of histones play significant roles in transcriptional regulation of eukaryotic cells. Several reports have been published on its role in gene expression (3). Histone acetylation and deacetylation are catalysed by specific enzyme families, histone acetyl-transferases (HATs) and deacetylases (HDACs) respectively. HATs were originally identified as transcriptional coactivators and HDACs as yeast transcriptional regulators. There are at least four groups of proteins with intrinsic HAT activity, while eight HDACs belonging to three distinct classes have been described in mammalian cells (2).

The balance between acetylation and deacetylation is an important factor in regulating gene expression and is thus linked to the control of cell fate (Fig. 1). Disruption of HAT or HDAC activity is possibly associated with cancer development (4). Genes that encode HAT enzymes are either translocated, or amplified, or overexpressed, or mutated in different types of cancers. Nucleosomes containing highly charged hypoacetylated histones bind tightly to the phosphate backbone of DNA, inhibiting transcription, presumably, because transcription factors, regulatory complexes, and RNA polymerase do not have access to DNA. Acetylation neutralizes the charge of the histones and generates a more open DNA conformation. Since aberrant histone acetylation has been linked to malignant diseases in some cases, HDAC inhibitors (HDIs) have potential as new drugs due to their ability to 
modulate transcription and to induce differentiation and apoptosis (5). Molecular analyses of human diseases have suggested that changes in acetylation may play a role in the uncontrolled cell growth of cancer.

The HDIs have been used as a new class of antineoplastic agents currently being evaluated in clinical trials. While these agents have been studied extensively in the laboratory, only recently has their mechanism of action begun to be elucidated. The field of HDIs is moving into a new phase of development. The exponential growth in the level of research activity surrounding the HDACs witnessed over the past decade has now started to produce success in the clinic, particularly in the field of oncology. Over the next few years experts believe that as first generation HDIs produce clinical benefits and second generation inhibitors are rationally designed with improved specificity, this class will emerge as a new class of cancer treatment.

We review knowledge on HDIs, the mechanisms of HDAC inhibition, and their role as a novel target of anticancer therapy, and the first clinical studies that have shown that histone hyperacetylation could be achieved safely in humans.

\section{HDAC inhibitors}

The classical group of HDIs include short chain fatty acids (such as 4-phenylbutyrate and valproic acid), hydroxamic acids (such as suberoylanilide hydroxamic acid or SAHA, pyroxamide, trichostatin A or TSA, oxamflatin and CHAPs), cyclic tetrapeptides (such as trapoxin, apicidin and depsipeptide-also known as FK-228 or FR 901228), benzamides (such as MS-275) and a variety of other chemical compounds (6-9). HDIs have been identified in natural sources, and synthetic inhibitors are also available (Table I).

Although reports on the activity and structure of the short chain fatty acids are limited, they often used as tools for studying HDACs in vitro and in clinical research (10). A widely prescribed anticonvulsant, valproic acid, acts as an HDI at relatively high concentrations (11). An antifugal agent, TSA, has relatively high reactivity and instability, and has been extensively used as a tool for the study HDAC function (12). A fungal metabolite, depudecin, that inhibits HDAC activity effectively both in vivo and in vitro has also been used (13).

SAHA and pyroxamide, are members of a class of HDAC inhibitors that are amenable to extensive structure-activity studies, and optimization of the SAHA structure has yielded compounds with subnanomolar activities (14). Among the more recently synthesized molecules belonging to this class of HDIs are the CHAP compounds, able to exert their inhibitory effect even at nanomolar concentrations $(15,16)$. It has also been shown that newly synthesized sulphonamide hydroxamic acid products were able, at micromolar concentrations, to exert antiproliferative action against human colon tumor cells in vitro (17).

Several cyclic tetrapeptides, such as apicidin and trapoxin, also inhibit HDACs at nanomolar concentrations. The apicidin $(18,19)$, interacts with the HDAC catalytic site with their ethyl ketone component, and the trapoxin (20), which irreversible inhibits HDACs interacting to their

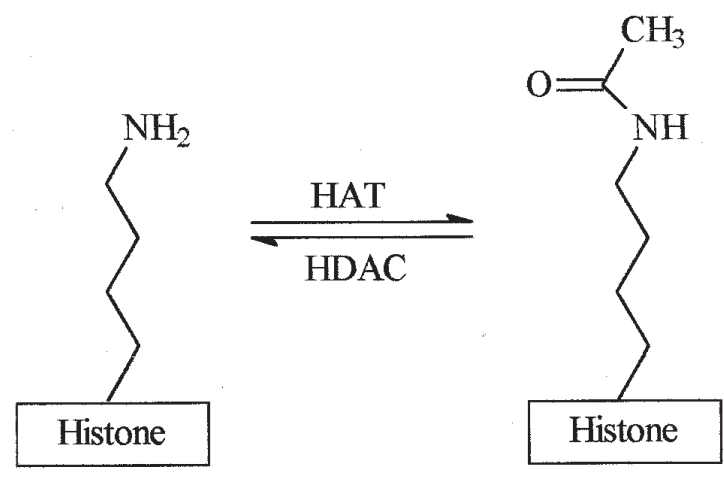

Figure 1. Acetylation and deacetylation of histones, catalyzed by histone acetyltransferase (HAT) and deacetylase (HDAC).

catalytic site. Depsipeptide $(7,21,22)$ is a natural product also able to inhibit HDACs in nanomolar concentrations. The fourth class of HDAC inhibitors consists of a structurally diverse group of agents that invariably contain a benzamide. This part of the molecule interacts with the catalytic site of HDAC, binding to zinc. Members of this class also include MS-275 that inhibits HDACs at micromolar concentrations and CI-994 (N-acetyldinaline), which seems to be an indirect HDI (23). In recent years, an increasing number of structurally diverse HDIs have been identified that inhibit proliferation and induce differentiation and/or apoptosis of tumor cells in cell lines and in animal models.

\section{Mechanisms of HDAC inhibition}

Acetylation disorders may be due to hyperacetylation and concomitant derepression of normally repressed promoters, leading to the presence of a set of proteins at an inappropriate moment. Conversely, underacetylation of a set of promoters could have equally deleterious effects by repressing expression of genes necessary for maintenance of a certain phenotype. In each of these cases, the balance of acetylation and deacetylation is disturbed. However, the actual situation encountered in vivo may be more complex, in that a misdirection of HAT or HDAC activity to wrong targets could be the disease-triggering process.

Inhibition of the HDACs is able to promote a variety of different anticancer mechanisms including apoptosis, cell differentiation and cell cycle inhibition (24). It is well known that histone acetylation is associated with gene transcription activation (25-27). HDIs target gene expression without modifying DNA sequence. They bind tightly to histones and prevent the transcription and expression of tumor suppressor gene (28). Although HDIs induce the expression of less than $2 \%$ of genes in cultured cells, there appeared to be some specificity to this effect (29). HDIs, such as phenylbutyrate, TSA, and SAHA induce the expression of CDKN1A gene, which encodes the cyclin-dependent kinase (Cdk)- inhibitor $\mathrm{p} 21^{\mathrm{WAF} 1}(30,31)$. The induction of $\mathrm{p} 21^{\mathrm{WAF} 1}$ and down regulation of cyclin A correlates well with the decreased CDK2 activity and cell cycle arrest. Transient transfection studies found that the induction of $\mathrm{p} 21$ by TSA requires the presence of the SP1 site in the promoter. This indicates that the HDI acts directly on the CDKNIA promoter, not on an upstream element of the pathway. 
Table I. Histone deacetylase inhibitors.

\begin{aligned} & 1 Apicidin \\ & 2 Butyrates \\ & 3 CI-994 (N-acetyl dinaline) \\ & 4 Depsipeptide (FR01228, FK-228) \\ & 5 Depudecin \\ & 6 m-Carboxy cinnamic acid bishydroxamic acid (CBHA) \\ & 7 MS-275 \\ & 8 Oxamflatin \\ & 9 Pyroxamide \\ & 10 Scriptaid \\ & 11 Suberoylanilide hydroxamic acid (SAHA) \\ & 12 TPX-HA analogue (CHAP) \\ & 13 Trapoxin B \\ & 14 Trichostatin A (TSA) \\ & 15 Valproic acid \\ & \hline\end{aligned}

Studies on the mechanism of cell growth inhibition by MS-275, showed that downregulation of c-myc mRNA levels and morphological reversion of H-ras transformed NIH 3T3 murine fibroblasts in culture, correlated with cell cycle arrest in G1 phase (32). MCF7 human breast adenocarcinoma cells are arrested in both $\mathrm{G} 1$ and $\mathrm{G} 2 / \mathrm{M}$ phases of the cell cycle (21). In a panel of human tumor cell lines treated with MS275 , induction was observed of $\mathrm{p} 21^{\mathrm{WAF} 1 / \mathrm{CIP} 1}$ in a $\mathrm{p} 53-$ independent manner and also downregulation of cyclin D1, which resulted in inhibition of CDK activity, hypophosphorylation of the retinoblastoma protein $\mathrm{Rb}$ and $\mathrm{G} 1$ arrest (33). Induction of $\mathrm{p} 21^{\mathrm{WAF} / \mathrm{CIP} 1}$ expression is thought to play an important if not deterninant role in the arrest of cell growth in G1. Cell clones lacking p21 were not arrested in G1 but continued DNA synthesis and were arrested in G2/M phase (33).

SAHA has been shown to induce accumulation of acetylated histones in the chromatin that is associated with the CDKN1A gene, and this increase correlates with increase of its transcription (34). About $2 \%$ of the expressed genes present alterations at their transcriptional level in TSA or SAHA treated transformed cells in culture (35). In addition, treatment with HDI attenuated the activation of $\mathrm{p} 38$ mitogenactivated protein kinases (MARK) and extracellular signalregulated kinase MARK, and increased the expression levels of cAMP induced apoptosis protein (cIAP)-1, suggesting that the observed increased adaptation and chromosomal instability induced by inhibiting HDAC activity might be directly connected with the activation of cell survival and/or antiapoptotic signals (36-38). TSA has also been shown to activate the $\mathrm{p} 15^{\mathrm{INK} 4 \mathrm{~B}}$ gene, a member of INK4 gene family, and induce transcription of the telomerase catalytic subunit, TERT, in several normal human cell types 39). Therefore, further studies are needed in order to determine the molecular basis of this apparent selectivity in gene expression induced by HDIs.
In human ER-negative breast cancer cell lines, 5-azacytidine (AZAC) alone induced 30-40-fold ER transcript; the addition of TSA elevated the ER mRNA expression about 10-fold more. The combination of AZAC and TSA induced a 300-400-fold increase in ER transcript (40). ER- $\alpha$ is a critical growth regulatory gene in breast cancer and its expression level is tightly linked to the prognosis and treatment outcome of breast cancer patients, as in breast epithelial cells the loss of ER- $\alpha$ expression is critical for cancer progression. It was shown that HDAC1 interacts with ER- $\alpha$ in vitro and in vivo and suppresses ER- $\alpha$ transcription activity, implying a role of HDAC1 in breast cancer progression by promoting cellular proliferation. In stable transfected MCF-7 clones overexpression of HDAC1 induced loss of ER- $\alpha$ and significantly increased cell proliferation and colony formation, compared to the control MCF-7 cells. Treatment of stable MCF-7 clones with TSA induced re-expression of ER- $\alpha$ mRNA and protein (41).

It has been demonstrated that HDIs inhibit angio-genesis. FK228 causes histone acetylation of angiogenesis factors, such as vascular endothelial growth factor (VEGF) and basic fibroblast growth factor (bFGF), and has a suppressive effect on their expression, which suggests that the effect on the expression of angiogenesis factors is important for the antitumor efficacy of FK228 (42-44).

Being able to target specific HDAC isoforms is likely to improve the therapeutic margin of this class of drug, however, it has highlighted the need to clarify the optimal profile of an HDAC isoform target. To date 11 HDAC isoforms have been identified. HDAC1 has been shown in a large number of studies to mediate cellular effects consistent with anticancer activity. Hence, selective inhibitors such as MS275 (HDAC1 selective) may be expected to have advantages over SAHA and FK228 which are non-selective inhibitors and are therefore more likely to produce adverse effects. Selective HDAC1 inhibition does not, however, appear to represent the optimal profile of activity. Using functional genomics to identify those HDACs that could play a major role in treating cancer, it has been suggested that HDAC8 may be another appropriate target for anticancer therapy (45). Further, it has been reported that HDAC8 inhibition induces growth arrest and apoptosis in human cancer cells but not in normal cells. Developing dual HDAC1/ HDAC8 inhibitors may therefore offer a useful approach and for this reason more information is awaited (45).

\section{HDIs and anticancer therapy}

HDIs have been shown to possess diverse biological activities. In vivo xenograft studies have demonstrated many of these agents to be effective in the inhibition of tumor growth. One of the attractions of HDIs is their ability to optimize other therapeutic approaches. HDIs are able to increase the efficacy of drugs such as retinoic acids, vitamin D analogues and PPAR- $\gamma$ ligands in cancer models (46). Already in the field of HDIs, an impressive body of data describes the ability of these molecules to modulate a wide variety of cellular functions, including cell differentiation, cell cycle progression, apoptosis, cytoskeletal modifications and angiogenesis. By targeting these key components of tumor 
proliferation, HDIs have the potential to occupy a strong position in the fast-moving cytostatic market. Two major reasons for which HDIs could play such a key role, are their ability to improve the efficacy of existing cytostatics (such as retinoids) and moreover, to target the transcription of specific disease-causing genes, conferring unprecedented therapeutic windows to cancer therapy for the first time $(24,47)$.

The existing treatment strategies are mostly limited to general HDIs, some of which have pleiotropic and/or unknown effects in addition to deacetylase repression. The first clinical studies have shown that histone hyperacetylation can be achieved safely in humans and that treatment of cancer with such agents seems to become possible. Preliminary results from clinical trials suggest that these agents are very promising. As long as phase II data is in agreement with the earlier stage data already available then HDAC inhibitors could represent one of the next classes of anticancer agent.

Several HDIs have shown impressive antitumor activity in vivo with remarkably little toxicity in preclinical studies and are currently in phase I or II clinical trials $(36,48)$. HDIs currently in clinical trials include the hydroxamic acids suberoylanilide hydroxamic acid (SAHA; Memorial SloanKettering Cancer Center/Aton Pharma Inc.) and pyroxamide (National Cancer Institute/Southern Research Institute), the benzamides MS-275 (NSC; Shering AG), CI-994 (acetyldinaline; Pfizer Inc.) and the butyrate derivative 4-PBA (sodium-4-phenylbutyrate; Johns Hopkins University) $(27,49)$. A number of carboxylates, including butyric and phenylbutyric acid are in clinical trials for cancer treatment alone and in combination with other agents $(48,50)$. Phenylbutyrate was one of the first HDIs to be tested in patients. A related compound of sodium phenylbutyrate, arginine butyrate, was recently evaluated in a phase I trial on metastatic colorectal cancers in combination with interleukin-2, but induced severe liver damage (51). A combination of phenylbutyrate and all-trans retinoic acid (ATRA), for example, induced histone hyperacetylation and complete remission in a case of highly resistant promyelocytic leukemia before relapse occurred after 7 months $(46,52)$. Butyrate and its analogs are also currently being evaluated as differentiating agents in prostate cancers. SAHA and pyroxamide have recently been used in clinical trials, the most advanced of which is SAHA (phase II). In patients with either solid tumors or Hodgkin's disease, tumor regression and improvement of symptoms were observed at doses not exerting any clinical toxicity. A phase I trial with depsipeptide revealed promising results in patients with T-cell lymphoma, and hyperacetylation of histones in primary lymphoma cells was associated with the clinical improvement of patients (27).

In a phase II study, patients with peripheral T-cell lymphoma (PTCL) or cutaneous T-cell lymphoma (CTCL) were treated with FK-228 (Piekarz R, et al: Proc Am Soc Clin Oncol 21: abs. 88, 2002). From nine evaluable patients with CTLC, there were seven partial responses, and two additional patients that are ongoing. TSA has been shown to have a transcriptional repression, demonstrating a possible treatment strategy for the cases of leukemia where mistargeting of HDAC activity is suspected. However, because of its toxicity, use of TSA as a treatment agent for leukemia is severely limited. TSA has also been shown to effectively inhibit squamous carcinoma cell proliferation and keratinocyte growth ex vivo. TSA-mediated arrest was found to be irreversible (53). Therefore, it is possible to envision a topical application of TSA in skin cancer, limiting some of the toxic side effects.

Several studies have reported a link between dietary fiber uptake and histone acetylation. The protective effects of high-fiber diet with regard to colon cancer development are known. A possible mechanism is the production of butyrate through colonic bacterial fermentation of fibers, which inhibits proliferation of colon cancer cell lines. Although the concentration of butyrate in the colonic lumen exceeds levels that inhibit proliferation of colon cancer cells in tissue culture, these concentrations still allow growth in the colonic crypt (54). A possible explanation, is that different dietary fibers may vary in their efficacy of increasing butyrate production $(55,56)$, and possibly, the sensitivity of adenocarcinoma cells to butyrate can differ, at least in a tissue culture system (57). Combination of fluorouracil, phenylbutyrate, indomethacin and interferon- $\gamma$ in treating patients with advanced colorectal cancer is now in process, and information regarding this clinical trial from National Cancer Institute, USA, can be found at the electronic address http://www.cancer.gov/cancer_information/.

The benzamide class, which is generally less potent than the corresponding hydroxamate and cyclic tetrapeptide classes, includes MS-275 and CI-994. MS-275 is currently in phase I clinical trials. CI-994 is an orally bioavailable compound that causes accumulation of acetylated histones, although is not able to inhibit HDAC activity in a direct fashion (23). Combination trials of CI-994 for non-small cell lung cancer with either capecitabine or together with carboplatin and paclitaxel in phase II studies are now in progress (27).

The above-mentioned studies indicate that the inhibition of HDAC activity leads to relaxation of the structure of chromatin associated with a specific set of programmed genes. The relaxed chromatin structure allows these genes to be expressed, which finally inhibit tumor cell growth. It seems that the most advanced agents include constituents from the small-molecule hydroxamate (SAHA, phase II) and cyclic peptide (FK-228, phase II) structural classes $(58,59)$. Promising results from these studies will continue to drive the exploration of HDAC function and inhibitor design.

\section{Conclusions}

Due to the combinatorial nature of the above mentioned agents and HDAC interaction, a large number of regulatory proteins is set to expand dramatically, and it is quite possible that targeting a selected complex could control the transcription of a single gene. Therefore, the challenge of the future is to attribute specific HDAC complexes to cellular function, and to identify molecules able to block the activity of these functions. Meeting this challenge is likely to produce therapies with efficacy and selectivity not currently possible with the existing cytostatics, and the ability to target the expression of selected genes usually reserved for biologics. 
The use of HDIs, probably in association with classical chemotherapy drugs or in combination with DNA-demethylating agents, could be promising for cancer patients. There are many unanswered questions regarding the optimal evaluation and utilization of HDIs for cancer therapy. At clinical level further studies are needed in order to delineate the optimal dosage and the duration of therapy. Further evaluation is needed to establish the clinical activity of combination therapy using HDIs with cytotoxic drugs or differentiation agents, and studies are underway to address these issues.

\section{References}

1. Kouraklis G: Progress in cancer gene therapy. Acta Oncol 38: 675-683, 1999.

2. Kouraklis G and Theocharis S: Histone deacetylase inhibitors and anticancer therapy. Curr Med Chem - Anti-cancer Agents 2: 477-484, 2002.

3. Zwiebel JA: New agents for acute myelogenous leukemia. Leukemia 14: 488-490, 2000.

4. Timmerman S, Lehrmann H, Popesskaya A and Harel-Bellan A: Histone acetylation and disease. Cell Mol Life Sci 58: 728-736, 2001.

5. Yoshida M, Furumai R, Nishiyama M, Komatsu Y, Nishino N and Horinouchi S: Histone deacetylase as a new target for cancer chemotherapy. Cancer Chemother Pharmacol 48: S20, 2001.

6. Bouchain C, Leit S, Frechette S, et al: Development of potential antitumor agents. Synthesis and biological evaluation of a new set of sulphonamide derivatives as histone deacetylase inhibitors. J Med Chem 46: 820-830, 2003.

7. Piekarz R and Bates S: A review of depsipeptide and other histone deacetylase inhibitors in clinical trials. Curr Pharm Des 10: 2289-2298, 2004.

8. Van Ommeslaeghe KEG, Brecx V, Papeleu P, Iterbeke K, Geerlings P, Tourwe D and Rogiers V: Amide analogues of TSA: synthesis, binding mode analysis and HDAC inhibition. Bioorg Med Chem Lett 13: 1861-1864, 2003.

9. Jeong MR, Hashimoto R, Senatorov VV, Fujimaki K, Ren M, Lee MS and Chuang DM: Valproic acid, a mood stabilizer and anticonvulsant, protects rat cerebral cortical neurons from spontaneous cell death: a role of histone deacetylase inhibition. FEBS Lett 542: 74-78, 2003.

10. Gore SD and Carducci MA: Modifying histones to tame cancer: clinical development of sodium phenylbutyrate and other histone deacetylase inhibitors. Exp Opin Investig Drugs 9: 2923-2934, 2000.

11. Phiel CJ, Zhang F, Huang EY, Guenther MG, Lazar MA and Klein PS: Histone deacetylase is a direct target of valproic acid, a potent anticonvulsant, mood stabilizer, and teratogen. J Biol Chem 276: 36734-36741, 2001.

12. Marks PA, Richon VM and Rifkind RA: Histone deacetylase inhibitors: inducers of differentiation and apoptosis of transformed cells. J Natl Cancer Inst 92: 1210-1216, 2000.

13. Miller TA, Witter DJ and Belvedere S: Histone deacetylase inhibitors. J Med Chem 46: 5097-5114, 2003.

14. Richon VM, Webb Y, Merger R, et al: Second generation hybrid polar compounds are potent inducers of transformed cell differentiation. Proc Natl Acad Sci USA 93: 5705-5708, 1996.

15. Furumai R, Komatsu Y, Nishino N, Khochbin S, Yoshida M and Horinouchi S: Potent histone deacetylase inhibitors built from trichostatin A and cyclic tetrapeptide antibiotics including trapoxin. Proc Natl Acad Sci USA 98: 87-92, 2001.

16. Komatsu Y, Tomizaki KY, Tsukamoto M, et al: Cyclic hydroxamic-acid-containing peptide 31 , a potent synthetic histone deacetylase inhibitor with anti-tumor activity. Cancer Res 61: 4459-4466, 2001.

17. Lavoie R, Bouchain G, Frechette S, et al: Design and synthesis of a novel class of histone deacetylase inhibitors. Bioorg Med Chem Lett 11: 2847-2850, 2001.

18. Murray PJ, Kranz M, Ladlow M, et al: The synthesis of cyclic tetrapeptoid analogues of the antiprotozoal natural product apicidin. Bioorg Med Chem Lett 11: 773-776, 2001.

19. Meinke PT, Colletti SL, Doss G, et al: Synthesis of apicidinderived quinolone derivatives: parasite-selective histone deacetylase inhibitors and antiproliferative agents. J Med Chem 43: 4919-4922, 2000.
20. Kijima M, Yoshida M, Sugita K, Horinouchi S and Beppu T: Trapoxin, an antitumor cyclic tetrapeptide, is an irreversible inhibitor of mammalian histone deacetylase. J Biol Chem 268: 22429-22435, 1993 .

21. Nakajima H, Kim YB, Terano H, Yoshida M and Horinouchi S: FR901228, a potent antitumor antibiotic, is a novel histone deacetylase inhibitor. Exp Cell Res 241: 126-133, 1998.

22. Su GH, Sohn TA and Ryu B: A novel histone deacetylase inhibitor identified by high-throughput transcriptional screening of a compound library. Cancer Res 60: 3137-3142, 2000.

23. Prakash S, Foster BJ, Meyer M, et al: Chronic oral administration of CI-994: a phase I study. Invest New Drugs 19: 1-11, 2001.

24. Garea-Villar A and Steller M: Histone deacetylase inhibitors: understanding a new wave of anticancer agents. Int J Cancer 112: 171-178, 2004.

25. Marks P, Rifkind R, Richon VM, Breslow R, Miller T and Kelly WK: Histone deacetylases and cancer: causes and therapies. Nat Rev Cancer 1: 194-202, 2001.

26. Gregory PD, Wagner $\mathrm{K}$ and Horz W: Histone acetylation and chromatin remodelling. Exp Cell Res 265: 195-202, 2001.

27. Roth SY, Denu JM and Allis CD: Histone acetyltransferases. Ann Rev Biochem 70: 81-120, 2001.

28. Garber K: Purchase of Aton spotlights HDAC inhibitors. Nat Biotechnol 22: 364-365, 2004.

29. Butler LM, Zhou X, Xu WS, Scher HI, Rifkind RA, Marks PA and Richon VM: The histone deacetylase inhibitor SAHA arrests cancer cell growth, up-regulates thioredoxin-binding protein-2, and down-regulates thioredoxin. Proc Natl Acad Sci USA 99: 11700-11705, 2002.

30. Saito A, Yamashita T, Mariko Y, et al: A synthetic inhibitor of histone deacetylase, MS-27-275, with marked in vivo antitumor activity against human tumors. Proc Natl Acad Sci USA 96: 4592-4597, 1999.

31. Vrana JA, Decker RH, Johnson CR, et al: Induction of apoptosis in U937 human leukemia cells by suberoylanilide hydroxamic acid (SAHA) proceeds through pathways that are regulated by Bcl-2/Bcl-XL, c-jun, and p21 CIP1, but independent to p53. Oncogene 18: 7016-7025, 1999.

32. Ueda H, Nakajima H, Hori Y, Goto T and Okuhara M: Action of FR901228, a novel antitumor bicyclic depsipeptide produced by Chromobacterium violaceum no 968, on Ha-ras transformed NIH3T3 cells. Biosci Biotech Biochem 58: 1579-1583, 1994.

33. Sandor V, Senderowicz A, Mertins S, Sackett D, Sausville E, Blagosklonny MV and Bates SE: P21-dependent G1 arrest with downregulation of cyclin D1 and upregulation of cyclin E by the histone deacetylase inhibitor FR901228. Br J Cancer 83: 817-825, 2000.

34. Richon VM, Emiliani S, Verdin E, Webb Y, Breslow R, Rifkind RA and Marks PA: A class of hybrid polar inducers of transformed cell differentiation inhibits histone deacetylases. Proc Natl Acad Sci USA 95: 3003-3007, 1998.

35. Huang L and Pardee AB: Suberoylanilide hydroxamic acid as a potential therapeutic agent for human breast cancer treatment. Mol Med 6: 849-866, 2000.

36. Vigushin DM and Coombes RC: Histone deacetylase inhibitors in cancer treatment. Anticancer Drugs 13: 1-13, 2002.

37. Cress WD and Seto E: Histone deacetylases, transcriptional control, and cancer. J Cell Physiol 184: 1-16, 2000.

38. Shin HJ, Baek KH, Jeon AH, et al: Inhibition of histone deacetylase activity increases chromosomal instability by the aberrant regulation of mitotic checkpoint activation. Oncogene 22: 3853-3858, 2003.

39. Hitomi T, Matsuzaki Y, Yokota T, Takaoka Y and Sakai T: P15 (INK4b) in HDAC inhibitor-induced growth arrest. FEBS Lett 554: 347-350, 2003

40. Yang X, Phillips DL, Ferguson AT, Nelson WG, Herman JG and Davidson NE: Synergistic activation of functional estrogen receptor (ER)-alpha by DNA methyltransferase and histone deacetylase inhibition in human ER-alpha-negative breast cancer cells. Cancer Res 61: 7025-7029, 2001.

41. Kawai H, Li H, Avraham S, Jiang S and Avraham HK: Overexpression of histone deacetylase HDAC1 modulates breast cancer progression by negative regulation of estrogen receptor alpha. Int J Cancer 107: 353-358, 2003.

42. Sasakawa Y, Naoe Y, Noto T, et al: Antitumor efficacy of FK228, a novel histone deacetylase inhibitor, depends on the effect on expression of angiogenesis factors. Biochem Pharmacol 66: 897-906, 2003. 
43. Kwon HJ, Kim MS, Kim MJ, Nakajima H and Kim KW: Histone deacetylase inhibitor FK228 inhibits tumor angiogenesis. Int J Cancer 97: 290-296, 2002.

44. Mie Lee Y, Kim SH, Kim HS, Jin Son M, Nakajima H, Jeong Kwon $\mathrm{H}$ and Kim KW: Inhibition of hypoxia-induced angiogenesis by FK228, a specific histone deacetylase inhibitor, via suppression of HIF-1 alpha activity. Biochem Biophys Res Commun 300: 241-246, 2003.

45. Peart M, Tainton KM, Ruelfi AA, et al: Novel mechanisms of apoptosis induced by histone deacetylase inhibitors. Cancer Res 63: 4460-4471, 2003

46. Theocharis S, Margeli A and Kouraklis G: Peroxisome proliferator activated receptor-gamma ligands as potent antineoplastic agents. Curr Med Chem - Anti-cancer Agents 3: 239-251, 2003.

47. Kelly WK, O'Connor OA and Marks PA: Histone deacetylase inhibitors: from target to clinical trials. Exp Opin Investig Drugs 11: 1695-1713, 2002.

48. Johnstone RW: Histone deacetylase inhibitors: novel drugs for the treatment of cancer. Nat Rev Drug Discov 1: 287-299, 2002.

49. Vigushin DM: FR-901228 Fujisawa/National Cancer Institute. Curr Opin Invest Drugs 3: 1396-1402, 2002.

50. Marks PA, Richon VM, Breslow R and Rifkind RA: Histone deacetylase inhibitors as new cancer drugs. Curr Opin Oncol 13: 477-483, 2001.

51. Douillard JY, Bennouna J, Vavasseur F, Deporte-Fety R, Thomare P and Giacalone F: Phase I trial of interleukin-2 and high dose arginine butyrate in metastatic colorectal cancer. Cancer Immunol Immunother 49: 56-61, 2000.
52. Warrell RP Jr, He L, Richon V, Calleja E and Pandolfi P: Therapeutic targeting of transcription in acute promyelocytic leukemia by use of an inhibitor of histone deacetylase. J Natl Cancer Inst 90: 1621-1625, 1998.

53. Saunders N, Dicker A, Popa C, Jones S and Dahler A: Histone deacetylase inhibitors as potential anti-skin cancer agents. Cancer Res 59: 399-404, 1999.

54. Csordas A: Butyrate, aspirin and colorectal cancer. Eur J Cancer Prev 5: 221-231, 1996.

55. McIntyre A, Gibson PR and Young GP: Butyrate production from dietary fibre and protection against large bowel cancer in a rat model. Gut 34: 386-391, 1993.

56. Zoran DL, Turner ND, Taddeo SS, Chapkin RS and Lupton JR: Wheat bran diet reduces tumor incidence in a rat model of colon cancer independent of effects on distal luminal butyrate concentrations. J Nutr 127: 2217-2225, 1997.

57. Stokrova J, Sovova V, Sloncova E and Korb J: Different morphological changes of two colorectal adenocarcinoma cell lines after sodium butyrates treatment. Int J Mol Med 4: 669-674, 1999.

58. Marshall JI, Rizvi N, Kauh J, et al: A phase I trial of depsipeptide (FR901228) in patients with advanced cancer. J Exp Ther Oncol 2: 325-332, 2002.

59. Sandor V, Bakke S, Robey RW, et al: Phase I trial of the histone deacetylase inhibitor depsipeptide (FR901228, NSC 630176), in patients with refractory neoplasms. Clin Cancer Res 8: 718-728, 2002. 